\title{
Optimized Smart Manufacturing Solution for Theory of Constraints based Efficiency Monitoring System (OTBEMS)
}

\author{
Venkataraman Balaji, P. Venkumar, M. S. Sabitha, S. Vijayalakshmi
}

\begin{abstract}
The purpose of every business organization is to improve profitability and retain its competitiveness. To achieve these manufacturing industries look for the latest tools and improvement methodologies to increase their effectiveness. Theory of Constraints (TOC) is one of the very popular and widely used improvement tools that view any manageable system as being limited in achieving more of its goals by a very small number of constraints. TOC uses a focusing process to identify the constraint and restructure the rest of the organization around it. Industry 4.0 takes the industries to the age of Cyber-physical systems. This paper provides an optimized smart manufacturing solution that derives the benefits of both TOC and IoT (Internet of Things) World. A new toolkit XBK (Extended bridge kit) is introduced in this system. XBK introduces flexibility in the IoT implementation. This experiment is conducted in a real-time test environment.
\end{abstract}

Keywords: About four key words or phrases in alphabetical order, separated by commas.

\section{INTRODUCTION}

$\mathrm{I}_{\mathrm{n}}$ ndustry 4.0 moves the business to the 4 th industrial revolution. It supports in the latest and technological way where the products, supportive procedures and people are associated through data can be collected, traced, extracted and examined. Industry4.0 supports industries to redesign their processes and system with the introduction of Internet of Things (IoT) in the traditional systems to deliver latest facilities like online and real-time monitoring and assistance.

Industrial IoT systems have helped the industries digitally transfer their processes by predicting required maintenance and the reduction of unplanned downtime by fixing the sensors and big data analytics and required algorithms. The usage of IoT will lead to cost savings, increased efficiencies, better quality and better market share over time.

Apart from various advantages, Industry 4.0 also produces

Revised Manuscript Received on December 05, 2019.

* Correspondence Author

Venkataraman Balaji *, President, TVS Sensing solutions private limited, Madurai, Research scholar, Kalasalingam academy of research and education, Krishnan Koil. Email: balaji.v@tvsss.co.in

Dr.P.Venkumar, Senior Professor, Department of Mechanical Engineering, Kalasalingam academy of research and education, Krishnan Koil. Email: p.venkumar@klu.ac.in

Dr.Sabitha.M.S, Head Informatiion Systems, TVS Sensing solutions private limited, Madurai. Email: sabimurali@rediffmail.com

Dr.S.Vijayalakshmi, Associate professor, Thiagarajar college of Engineering. Email: svlcse@tce.edu the latest technology challenges for the industries during the implementation time. In this case study, an additional focus is given to the old machines which are not capable of providing the machine status and its efficiency by default. The days where man uses to guide machines are gone, and now the reverse is happening in every field. The IoT application discussed in this paper is used for monitoring and controlling the machines with the help of sensors, enhancing its operational effectiveness and creating a real-time online system. A cyber environment is obtained with the latest technologies as the production results are obtained with high accuracy and calibration without any manual errors.

In recent years, many systems have been developed using IoT in the manufacturing environment. V.Balaji et al., [6] suggested a Sensor-Based (SBEMS) model where appropriate sensors are used in every process on the shop floor in combination with the cloud improves the productivity and mitigates the errors in the manufacturing plant. Even though it brings out intelligent insights, the implementation cost is higher in large industries.

To mitigate the problems in the SBEMS model, Balaji et al., [3] suggested an innovative TBEMS system. In TBEMS, the TOC (Theory of Constraints) principle is applied in the shop-floor reduces the implementation cost, network congestion, connectivity and data related issues. In this model, bottleneck operations were identified using the TOC principle. Then the SBEMS system was incorporated only for the identified bottleneck operations. This approach combines the best of TOC and IoT world. The application of IoT exploitation of better productivity and effective installation for constrains alone ensures the smartness of the system.

Even though the TBEMS system extracted the benefits of SBEMS, it costs more when the bottleneck operation and the priority changes in the shop-floor. Over a period of time, the bottleneck operation changes when some other process fails to meet customer demand. In such case, the SBEMS implementation of the new bottleneck operations increases the cost and other issues.

The proposed XBEMS system is designed in such a way that the Dynamic connectivity is implemented. This approach increases the efficiency of the manufacturing system, enhances the profit, reduces the operation expense and improves the outcome.

In this paper, Section 2 briefs the previous works on 
smart manufacturing systems, section 3 describes the structure and functionality of smart manufacturing through Sensor Based Efficiency Monitoring Systems (SBEMS), and Toc Efficiency Monitoring Systems (TBEMS). The proposed OTEBMS system discussed in section 4 and the results were discussed in section 5 .

\section{PRevious WORK}

IoT is capable of decision making, controlling the environment and for providing additional services [1]. Augusto Ciuffoletti et al., [2] approached a low-cost IoT in a holistic manner. It proposed a conceptual framework for cost evaluation and its effectiveness was verified. A prototype sensor was designed and tested with its surrounding eco-system. With these results, performance and cost for a pilot system made of fifty sensors, which were deployed in an urban area were estimated. It ensures an affordable investment even for a rural community.

Eli goldratt popularized the concept of ToC (theory of constraints). Using TOC, Balaji.v et al., [3] suggested TBEMS (ToC based efficiency monitoring system) that involves a lesser number of sensor connectivity, lesser cost, lower resource utilization, simple computational methodologies and simple database management. The TBEMS model can be attained by identifying bottleneck operations using ToC and installation of IoT set up in all the selected machines. On blending the concept of ToC with IoT, the implementation speed can be augmented for quick results.

Shabir Ahmad et al., [4] presented a comparative analysis of existing simulators with visualization technologies that assisted in the feasibility analysis of real-time input tasks for IoT embedded applications. For interfacing and visualizing, many groundwork adopt Web-based technologies. The virtual simulator built using web based technologies are platform independent providing worldwide access. It has been found that live streaming and shared resources are some of the attributes, the simulator that is desired to have in a tool. Most of the tools that were studied lack the above mentioned attributes were rectified using the algorithms rate monotonic (RM) and earliest deadline first (EDF).

Stefano tedeschi et al, [5] proposed an approach for IoT modular architecture implementation in legacy system. It is tedious to monitor and control legacy machine tools that are often isolated, not well-equipped with modern communication technologies in the entire production process. The author discussed that it is necessary that instantaneous decisions are to be made for smart manufacturing at the management level, considering aspects such as complexity, speed, and security. The Rule Based Fuzzy Cognitive Maps (RBFCM) technique, assess the pros and cons of the legacy machines and gives a high-level conception for IoT configuration according to targets and cost in an efficient manner.

V.Balaji et al., [6] proposed a sensor based efficiency monitoring system (SBEMS) which leads to smart manufacturing and has the benefits of predicting and preventing the potential failures well before it occurred, the storage of quality data for its future retrieval and traceability, effective compensation for the loss time and usage of cloud for connectivity with internet service in the shop floor. In spite of supervising manpower efficiency using several manual systems, SBEMS differs by alerting the decision-makers online without entering the data into the systems.

From various studies and research it is clear that for the implementation of smart manufacturing systems, there must be due consideration of the implementation cost and the return on investment which leads to the proposal of OTBEMS model.

\section{EXISTING SBEMS AND TBEMS}

\section{A. SBEMS}

SBEMS is a simplified approach initiated with the IoT model on the shop floor. This is a cost-competitive, low energy consumption model bringing out cyber-physical system. Collection of data and normal analytics tool are used to mine the insights. It ensures data processing to the best availability of data and proactive solution for issues. A sensor when being attached to various machines gets triggered by the actions of machines and converts the physical parameters to electrical signals.

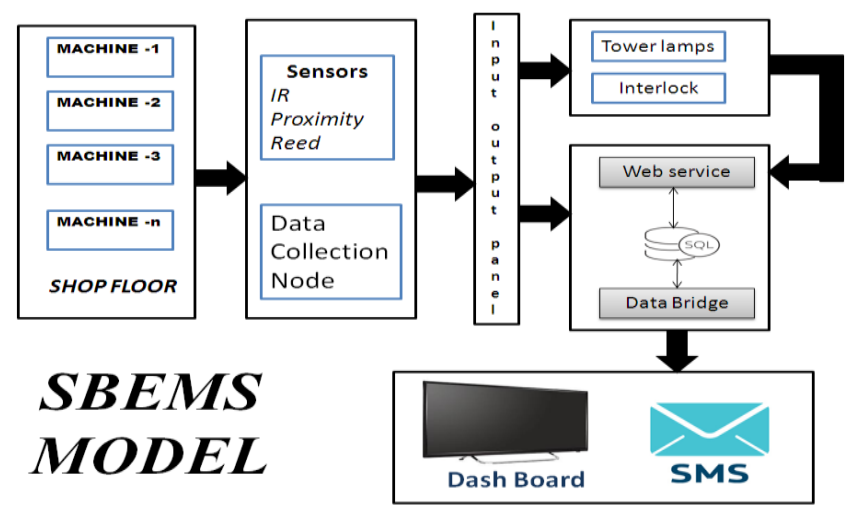

Fig.1. SBEMS Model

Depending on the process executed by the machine, different types of sensors are chosen to sense the activity. These sensors help the machines to report to the I/O panel that digitalizes the signal. The collected data alerts with the help of tower lamps and interlocks at the time of emergency. Even SMS is sent to the respected person by web-based monitoring systems. The system has the benefits of predicting and preventing the potential failures well before its occurrence, the storage of quality data for its future retrieval and traceability, effective compensation of the loss time and usage of cloud for connectivity with internet service on the shop floor.

The proposed system does not address all the parameters for OEE (overall equipment efficiency) calculation. Data integrity, data confidentiality and data ownership are not satisfied. Storage and power problems, connectivity issues, congestion, network traffic issues can be benefitted by IoT setup with some 
optimization done during the implementation process.

\section{B. TBEMS}

TBEMS model is the extension of SBEMS model. The ToC principle is applied in SBEMS to identify the bottleneck process on the shopfloor. ToC seeks popularity in a production environment and bottleneck operations are identified using drum buffer rope. It emphasizes that constraint resources alone would impact on global outcome signifying that local outcome is not the same as global outcome. The IoT implementation can be extended only for the bottleneck operations. A simple block diagram of the TBEMS model is given in Figure 2.

TBEMS MODEL

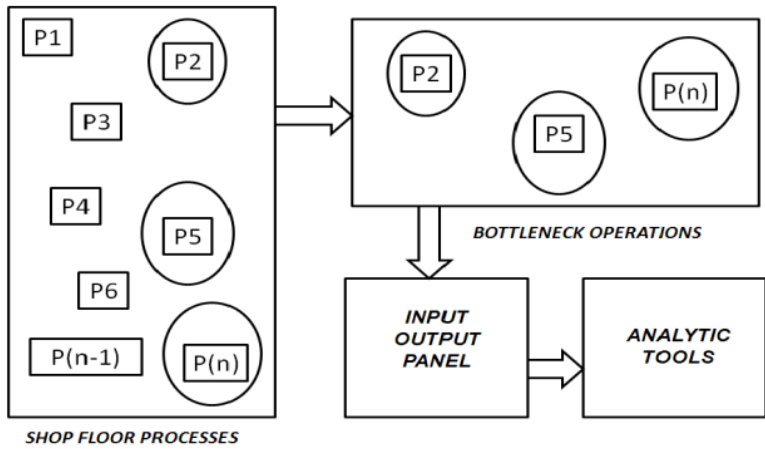

Fig. 2.TBEMS model

The TBEMS experiment has been conducted in a real-time test environment. The experiment results proved that the efficiency is improved in the data and network management at a lower cost.

As the sensor connectivity in the TBEMS model is less, server costs will also become lower. It also lessens energy in terms of cost, computational methodologies, data administration and lower resource utilization and easy database management. If in any of the bottleneck operation identification steps, a constraint has been broken, the identification of bottleneck operation must begin from first due to which there is wastage of resources and an increase in implementation cost. Adding reusability of resources to TBEMS, an IoT setup for the real manufacturing system is proposed.

\section{Problem Description}

The aim of the manufacturing companies is to provide quality products in the stipulated time frame. In TBEMS model, IoT Technology is extended only to the bottleneck operation identified using ToC on the production shop floor. This helps in monitoring the efficiency of the product.

Over a period of time, while applying ToC on the shop floor, some other process may become bottleneck and the previous bottleneck process may set right after applying associated process improvements. Then the focus point changes from the old bottleneck to the current bottleneck operation. In TBEMS, the IoT facility needs to be extended to the new bottleneck operation. The following problems with the TBEMS need to be addressed again when the number of bottleneck operation increases
- Sensor and address related issues

- Network related problems

- Congestion Control

- Data buffers

- Network traffic and overloading

- Selection of relevant Software and Algorithm

- Storage and power problem

- Connectivity issues

Although exploiting the best of ToC, it fails to satisfy the needs in some cases. Whenever the bottleneck operation satisfies the constraints or sometimes when the other processes break down the requirements, the TBEMS system is depleted. All the steps must be repeated from the beginning that leads to a huge implementation loss and declining the feature of reusability. The proposed system suggests a dynamic implementation of connectivity. The system aims to define connectivity to the processes at the required instant that is when they are identified as bottleneck operation without repeating all the steps in TBEMS. Thus, the implementation cost is reduced.

A new optimized solution OTBEMS (Optimized Toc Based efficiency monitoring system) proposed in this paper to address the above problems.

\section{PROposed OTBEMS}

The proposed OTBEMS model is the extended version of SBEMS model and utilizes the benefits of TBEMS model. The advantage of TBEMS model is to extend the SBEMS model only for the bottleneck operations identified by the TOC principle. When the bottleneck operation changes from one process to any other process, the SBEMS need to be extended for several stations and whatever problems identified before installing the TBEMS model remains the same. This is again back to SBEMS even though we apply TOC principle.

This model introduces an XBK (Extended bridge kit). This is a small device that can connect any three machines at a time. If the bottleneck operation changes from one process to another, the connectivity with that machine needs to be changed in the previous model. The XBK kit helps to move the connectivity from machine to machine easily. This kit acts as a plug and play model of bridging the machine with the kit. The model of XBK is given in Figure 3.

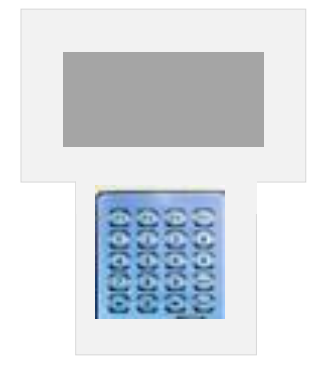

Fig.3. Extended bridge kit (XBK) 
The design of the XBK kit is simple, scalable, configurable, IIoT (Industrial Internet of Things) based and Web-ready. The following are the technical features of the XBK kit.

- 15 to $24 \mathrm{~V}$ Supply

- 10 mbps Ethernet

- 3.2" 128X64 Monochrome Display

- Optional Fingerprint for Authentication

- Optional RFID

- Optional 1kV RS232/RS485 (Modbus) (Max Baud rate 19200)

- Optional USB Host for USB keyboard/ barcode scanner

- Optional Wi-Fi

- Optional RTC

- I/O interfaces

- AI - 4 (4-20mA)

- DI - 4 (NPN)

- DO - $6(24 \mathrm{~V}, 500 \mathrm{~mA}$, total current $<2 \mathrm{~A})$

The block diagram of the OTEBMS model is depicted in Figure 4. In OTBEMS model, the implementation methodology is simplified and new methodology can be adopted easily even if the bottleneck operation changes. The entire hardware setup need not be replaced with the help of XBK.

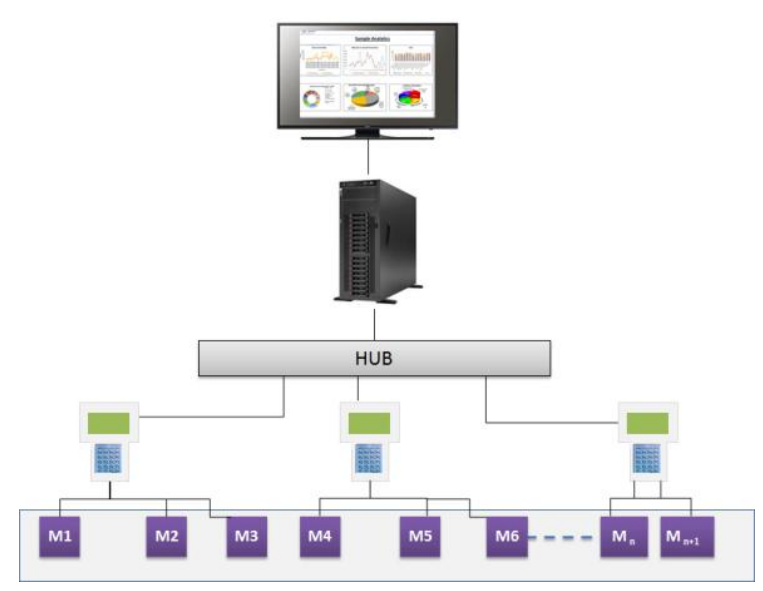

Fig.4. Architecture of OTBEMS

The bottom-most layer in the block diagram represents various machines on the shop floor. Three machines can be connected at a time with the XBK device at a time. This does not require any separate I/O panel. Everything is integrated into the XBK itself. The XBK further connected with the hub and the hub is directly connected to the server for data transfer. The data collected in the server further processed and data analytics have been carried out. The operators log into the system by using the employee id or fingerprint authentication. The job card of the job is synchronized with the existing ERP system.

Figure 5 indicates connectivity between the bottleneck operations and the XBK kit. The introduction XBK kit only for the bottleneck operations addresses the following issues of implementing IoT for the entire shop floor.

- Sensor and address related issues

- Network related problems

- Congestion Control

- Data buffers

- Network traffic and overloading

- Selection of relevant Software and Algorithm

- Storage and power problem

- Connectivity issues

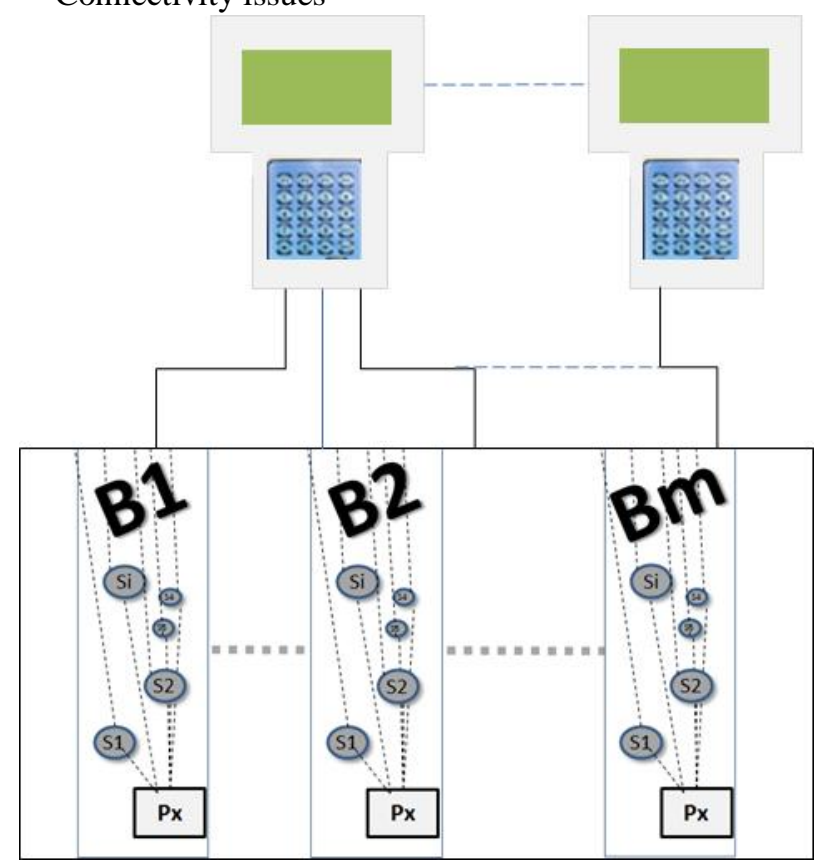

Fig.5. Bridging the bottleneck operations and XBK

In the proposed OTBEMS model, the network setup, I/O panel and related hardware setup are minimized with the selection of bottleneck operations. The bottleneck operation identified processes are represented as Px. The $\mathrm{x}$ varies from 1 to $\mathrm{n}$.

Let $\mathrm{Sc}$ - total cost involved for sensors, $\mathrm{Si}$ - Implementation cost of sensors, $\mathrm{Nc}$ - Network related implementation cost Uc - Implementation cost for a single machine

$$
U c=\sum_{\mathrm{s}=1}^{\mathrm{i}}(\mathrm{Sc}+\mathrm{Si}+\mathrm{Nc})
$$

$\mathrm{Uc}$ is the implementation cost for a single machine.

In SBEMS model, the entire cost for all the process involved for implementation. SIc - SBEMS Implementation cost STc - Standard setup cost.

$$
S I c=\sum_{(p=1)}^{n}(U c)+S T c
$$

In (2), the SBEMS implementation cost is calculated for all the processes. The standard setup cost like network connection cost, server for data collection and data analytics is represented in STc.

In TBEMS model, the cost for selected bottleneck operation involved for implementation. TIc - TBEMS Implementation cost

OTc - Optimised setup cost (it involves additional cost

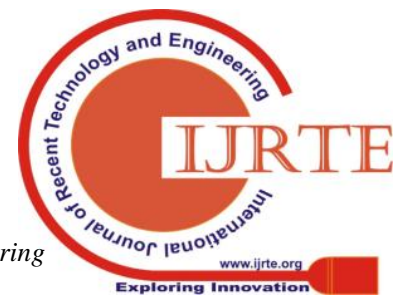


only for the bottleneck operations).

$$
T I c=\sum_{\mathrm{b}=1}^{\mathrm{n}}(\mathrm{Uc})+\mathrm{OTc}
$$

TIc $\alpha$ number of bottleneck processes.

In (3), the TBEMS implementation cost is calculated for the selected bottleneck processes. The standard setup cost like network connection cost, server for data collection and data analytics. This is optimized (OTc) in the case of TBEMS due to the selection of bottleneck operations.

The cost-saving with the TBEMS/SBEMS model is

$$
\begin{aligned}
& F c=\sum_{\mathrm{P}=1}^{\mathrm{n}}(\mathrm{SIc})-\sum_{\mathrm{B}=1}^{\mathrm{m}}(\mathrm{TIc})+S d \\
& S d=S T c-O T c
\end{aligned}
$$

After a few implementations, when the bottleneck operation changes, the implementation activities carried over the previous process can't be reused. Hence Fc $=0$ even though $\mathrm{Fc}$ is higher at the initial stage. Whatever is claimed as cost-benefit at the initial stage may not be same all the time. In the proposed OTBEMS model, the implementation cost the XBK involves network cost, Input/Output panel cost. Sensor fitment is applicable only for the bottleneck operations. Server and data analytics related costs are common for all the systems. The OIc (OTBEMS implementation cost) can be expressed as $\mathrm{Xc}-\mathrm{XBK}$ cost

$$
O I c=\sum_{\mathrm{b}=1}^{\mathrm{n}}(\mathrm{Sc}+\mathrm{Si}+\mathrm{Xc})
$$

Xc covers the cost of $<=3$ bottleneck operations. If the no. of bottleneck operations $>3, \mathrm{Xc}=2 * \mathrm{Xc} \sum_{\mathrm{B}=1}^{\mathrm{m}}(\mathrm{OIc}) \prec \sum_{\mathrm{B}=1}^{\mathrm{m}}$ (TIc) due to its reusability nature.

The proposed optimized OTBEMS model consumer lesser resources, lower cost, enhanced computational methodologies and simple database management.

\section{RESULTS AND DISCUSSION}

This experiment has been conducted in a real-time shop floor. The results of SBEMS, TBEMS and OTBEMS are compared in this section.

\section{A. Resource utilization}

The total implementation cost for the resources has been calculated with the formula (2),(3) and (6). This experiment is conducted in a product line which includes 11 processes and 3 processes were identified as a bottleneck operation.

Four iterations have been conducted in this experiment. The resource utilitisation varies for SBEMS, TBEMS and OTBEMS model. The total IoT implementation cost of Iteration 1,2,3 and 4 for various models are given in Figure 6.

\begin{tabular}{|c|c|c|c|c|c|c|c|c|c|}
\hline Resource & Iteration & $\begin{array}{l}\text { No. of } \\
\text { bottleneck } \\
\text { processes }\end{array}$ & Process & SBEMS qty & $\begin{array}{l}\text { SBEMS } \\
\text { cost }\end{array}$ & $\begin{array}{l}\text { TBEMS } \\
\text { qty }\end{array}$ & $\begin{array}{l}\text { TBEMS } \\
\text { cost }\end{array}$ & $\begin{array}{l}\text { OTBEMS } \\
\text { qty }\end{array}$ & $\begin{array}{l}\text { OTBEMS } \\
\text { cost }\end{array}$ \\
\hline \multirow[t]{4}{*}{ Sensors } & I & 3 & $\mathrm{P} 5, \mathrm{P} 9, \mathrm{P} 10$ & 33 & 82500 & 9 & 22500 & 9 & 22500 \\
\hline & II & 3 & P6, P9,P10 & 33 & 82500 & 9 & 30000 & 9 & 22500 \\
\hline & III & 3 & P6, P8,P10 & 33 & 82500 & 9 & 37500 & 9 & 22500 \\
\hline & IV & 4 & $\mathrm{P} 4, \mathrm{P} 6, \mathrm{P} 8, \mathrm{P} 10$ & 33 & 82500 & 9 & 45000 & 9 & 22500 \\
\hline \multirow[t]{4}{*}{ HMI unit } & I & 3 & $\mathrm{P} 5, \mathrm{P} 9, \mathrm{P} 10$ & 11 & 275000 & 3 & 75000 & 3 & 75000 \\
\hline & II & 3 & P6, P9,P10 & 11 & 275000 & 3 & 75000 & 3 & 75000 \\
\hline & III & 3 & P6, P8,P10 & 11 & 275000 & 3 & 75000 & 3 & 75000 \\
\hline & IV & 4 & P4,P6, P8,P10 & 11 & 275000 & 3 & 75000 & 3 & 75000 \\
\hline \multirow[t]{4}{*}{$\begin{array}{l}\text { Network } \\
\text { connectivity }\end{array}$} & I & 3 & $\mathrm{P} 5, \mathrm{P} 9, \mathrm{P} 10$ & & 25000 & & 7000 & & 1500 \\
\hline & II & 3 & P6, P9,P10 & & 25000 & & 9654 & & 1500 \\
\hline & III & 3 & P6, P8,P10 & & 25000 & & 10500 & & 1500 \\
\hline & IV & 4 & P4,P6, P8,P10 & & 25000 & & 12500 & & 1500 \\
\hline
\end{tabular}

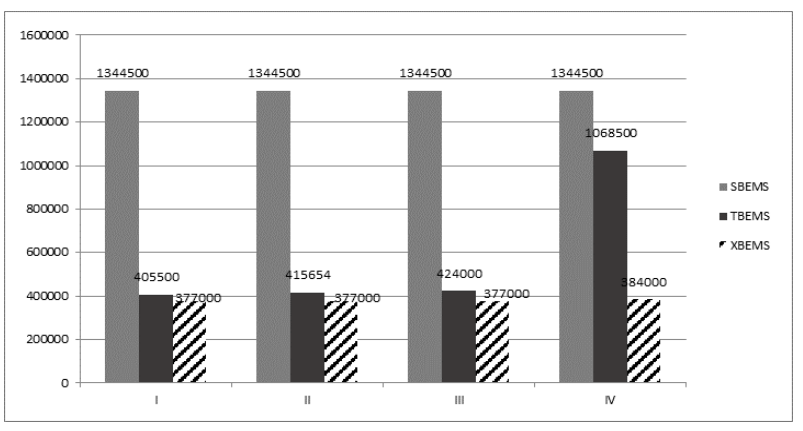

Fig.6. Implementation cost comparision chart

Table- I: Resource utilisation for the existing and proposed OTBEMS model 


\begin{tabular}{|c|c|c|c|c|c|c|c|c|c|}
\hline \multirow[t]{4}{*}{ I/O Panels } & I & 3 & P5, P9,P10 & 2 & 40000 & 1 & 30000 & -- & -- \\
\hline & II & 3 & P6, P9,P10 & 2 & 40000 & 1 & 30000 & & -- \\
\hline & III & 3 & P6, P8,P10 & 2 & 40000 & 1 & 30000 & & -- \\
\hline & IV & 4 & P4,P6, P8,P10 & 2 & 40000 & 1 & 30000 & & --- \\
\hline \multirow[t]{3}{*}{ Server } & I & 3 & P5, P9,P10 & 1 & 900000 & 1 & 265000 & 1 & 265000 \\
\hline & III & 3 & P6, P8,P10 & 1 & 900000 & 1 & 265000 & 1 & 265000 \\
\hline & IV & 4 & $\mathrm{P} 4, \mathrm{P} 6, \mathrm{P} 8, \mathrm{P} 10$ & 1 & 900000 & 1 & 900000 & 1 & 265000 \\
\hline \multirow[t]{4}{*}{ Tower lamp } & I & 3 & P5, P9,P10 & 11 & 22000 & 3 & 6000 & 3 & 6000 \\
\hline & II & 3 & P6, P9,P10 & 11 & 22000 & 3 & 6000 & 3 & 6000 \\
\hline & III & 3 & P6, P8,P10 & 11 & 22000 & 3 & 6000 & 3 & 6000 \\
\hline & IV & 4 & P4,P6, P8,P10 & 11 & 22000 & 3 & 6000 & 3 & 6000 \\
\hline \multirow[t]{4}{*}{ XBK } & I & 3 & P5, P9,P10 & -- & -- & -- & -- & 1 & 7000 \\
\hline & II & 3 & P6, P9,P10 & -- & -- & -- & & 1 & 7000 \\
\hline & III & 3 & P6, P8,P10 & -- & -- & -- & & 1 & 7000 \\
\hline & IV & 4 & P4,P6, P8,P10 & -- & -- & -- & & 2 & 14000 \\
\hline \multirow[t]{4}{*}{ Total Cost } & I & 3 & P5, P9,P10 & & 1344500 & & 405500 & & 377000 \\
\hline & II & 3 & P6, P9,P10 & & 1344500 & & 415654 & & 377000 \\
\hline & III & 3 & P6, P8,P10 & & 1344500 & & 424000 & & 377000 \\
\hline & IV & 4 & $\mathrm{P} 4, \mathrm{P} 6, \mathrm{P} 8, \mathrm{P} 10$ & & 1344500 & & 1068500 & & 384000 \\
\hline
\end{tabular}

\section{B. Data Inflow}

In this experiment, the sensor data collected from various machines depend on the number of machines connected to it. Each machine is connected with two sensors

This experimental setup liberates 3 sensor data from each machine for every millisecond. It liberates data only when the machine is in the ON condition. The data inflow is monitored in the TBEMS system and the complete IoT set up stations. The data inflow will vary depends on the availability of the machines. The number of records generated is listed in Table 2.

Table-II: Data inflow of SBEMS, TBEMS and OTBEMS

\begin{tabular}{|l|r|r|r|r|}
\hline $\begin{array}{c}\text { Number of } \\
\text { records in Nos } \\
\text { Lacs }\end{array}$ & 1 day & 7 days & 15 days & 20 days \\
\hline SBEMS & 3168 & 21200 & 45030 & 620500 \\
\hline TBEMS & 860 & 5887 & 12286 & 168227 \\
\hline OTBEMS & 860 & 5887 & 12286 & 168227 \\
\hline
\end{tabular}

In Figure 7, Data inflow is monitored for the entire IoT setup (SBEMS) and TBEMS model of implementation. It clearly indicates that the data inflow is much lower in the TBEMS model. Due to the lesser data inflow, this model will consume less energy, space and computational time for all kinds of database-related activities like query processing, storage and analytics.

\begin{tabular}{|c|c|c|c|c|}
\hline $\begin{array}{l}800000 \\
600000\end{array}$ & SB & del & \multicolumn{2}{|c|}{620500} \\
\hline 200000 & & 200 & 45030 & 168227 \\
\hline \multirow[t]{2}{*}{$2-0000$} & ${ }^{3168} 860$ & 5887 & 12286 & \\
\hline & 1 day & 7 days & 15 days & 20 days \\
\hline
\end{tabular}

Fig.7. Data inflow comparison

\section{CONCLUSION}

Industrial 4.0 helped the industries to move to the digital world. Even though the benefits of the SBEMS model are utilized in TBEMS, it costs more when the priority of the bottleneck operation changes in the process. The proposed OTBEMS has introduced an XBK kit that takes care of the lesser implementation. From the experiment, it is evident that it consumes lower cost when the bottleneck operation changes.

\section{REFERENCES}

1. Addressing ROI in Internet of Things Solutions, A practical approach to calculating the return on investment for the Internet of Your Things. Microsoft corporation, September 2016

2. A.Ciuffoletti, "Low-cost IoT: A holistic approach." Journal of Sensor and Actuator Networks 7, no. 2 (2018), p. 19.

3. V.Balaji, P.Venkumar, and M.S.Sabitha. "Smart Manufacturing through TOC based Efficiency Monitoring System (TBEMS)." International Journal of Engineering \& Technology 7, no. 4.10 (2018), pp. 118-123.

4. S.Ahmad, S.Malik, and D.H.Kim. "Comparative analysis of simulation tools with visualization based on real-time task scheduling algorithms for iot embedded applications." Int. J. Grid Distrib. Comput 11 (2018), pp. $1-10$.

5. S.Tedeschi, D.Rodrigues, C.Emmanouilidis, J.Erkoyuncu, R.Roy, \& A.Starr, "A cost estimation approach for IoT modular architectures implementation in legacy systems." Procedia Manufacturing 19 (2018), pp. 103-110.

6. V.Balaji, P. Venkumar, M.S.Sabitha, S.Vijayalakshmi, and RM Rathikaa Sre. "Smart manufacturing through sensor based efficiency monitoring system (SBEMS)." In International Conference on Soft Computing and Pattern Recognition, pp. 34-43. Springer, Cham, 2016. 


\section{AUTHORS PROFILE}

Venkataraman Balaji, is currently serving as President at TVS Sensing Solutions Private Limited. He received his B.E., and M.E degrees from Thiagarajar College of Engineering, Madurai, Tamilnadu, India in 1991 and 1997 respectively and pursuing Ph.D at Kalasalingam academy of Research and Education, India. His major areas of research interest are Strategy, Industrial Engineering, Industrial Internet of Things and Big Data.

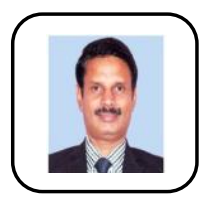

Dr. P. Venkumar, is currently a Senior Professor in Department of Mechanical Engineering, Kalasalingam Academy of Research and Education, Krishnankovil, Tamilnadu, Inida. He received his B.E., and M.E., degrees in Thigagrajar College of Engineering, Madurai, Tamilnadu, India, in 1994 and 1997, respectively and Ph.D. degree in Manonmaniam Sundaranar University, India in Industrial Engineering in 2006. His main areas of research interest are Manufacturing system optimization, Supply chain management and Smart manufacturing

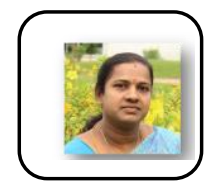

Dr.M.S.Sabitha is serving as Head, Information Systems at TVS Sensing Solutions Private Limited. She received her B.Sc and M.C.A from Madurai Kamaraj Univeristy in 1994 and 2000 respectively. M.Phil from Alagappa University in 2004 and Ph.D in Computer Science from Bharathiar University in 2019. Her main areas of research interest are Big data, Data mining and Internet of things.

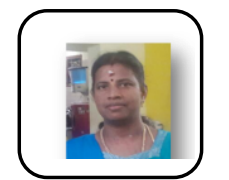

Dr.S.Vijayalakshmi is serving as an Associate professor at Thiagarajar College of Engineering. She received her M.Com from Madurai Kamaraj University in 1991 and M.C.A from Thiagarajar College of Engineering in 1994. M.Phil from Madurai Kamaraj University in 2004. Ph.D in Computer Science from Anna University in 2011. Her main areas of research interest are Big data and Data mining. 